\title{
ORTHOGONALITY AND NONLINEAR FUNCTIONALS
}

\author{
BY S. GUDDER AND D. STRAWTHER
}

\author{
Communicated by Robert Bartle, December 12, 1973
}

Let $X$ be a vector space of functions. In practice, $X$ is taken to be (i) the set of continuous functions on some type of topological space or (ii) a set of measurable functions on a measure space. We say that $x, y \in X$ are orthogonal in the lattice theoretic sense $\left(x \perp_{L} y\right)$ if $\{t: x(t) y(t) \neq 0\}$ is $\varnothing$ in case (i) or of measure zero in case (ii). A real valued functional $f: X \rightarrow R$ is $L$-additive if $f(x+y)=f(x)+f(y)$ whenever $x \perp_{L} y$. If $f$ is $L$-additive and satisfies certain continuity or boundedness conditions then $f$ admits an integral representation giving a nonlinear generalization of the Riesz theorem. Such representations have been obtained for case (i) in $[1],[4]$ and for case (ii) in [2], [3], [10], [11], [12], [14]. Although the above orthogonality is important for certain applications [5], [9], [13], in this note we consider orthogonalities which are standard in inner product and normed spaces. To some extent our results are less general than those in the above papers since the standard orthogonality is weaker than lattice theoretic orthogonality. On the other hand, some of our theorems apply to more general vector spaces than the above and furthermore we have obtained results for a more general class of functionals which we call orthogonally monotone functionals. Finally, we use our results to solve a nonlinear functional equation and give an application for the solution.

An orthogonality vector space is a real vector space $X$ with $\operatorname{dim} X \geqq 2$ on which there is defined a relation $x \perp y$ such that

(01) $0 \perp x, x \perp 0$ for all $x \in X$,

(02) if $x \perp y$ and $x, y \neq 0$ then $x, y$ are linearly independent,

(03) if $x \perp y$ then $\alpha x \perp \beta y$ for all $\alpha, \beta \in R$,

(04) if $B$ is a two-dimensional subspace of $X$, then for every $0 \neq x \in B$, there exists $0 \neq y \in B$ such that $x \perp y$ and $x+y \perp x-y$.

It is easily seen that any real vector space of dimension $\geqq 2$ is an orthogonality vector space if we define $0 \perp x, x \perp 0$ for all $x$ and if $x, y \neq 0$ then $x \perp y$ iff $x, y$ are linearly independent. It is also clear that an inner product space is an orthogonality vector space under the standard orthogonality

AMS (MOS) subject classifications (1970). Primary 46B99, 46C05, 47H15; Secondary $46 \mathrm{~F} 05,81 \mathrm{~A} 12$. 
relation. Furthermore, any normed space is an orthogonality vector space under the James [8] orthogonality relation.

Let $(X, \perp)$ be an orthogonality vector space. A functional $f: X \rightarrow R$ is hemicontinuous iff $\alpha_{i} \rightarrow \alpha$ implies $f\left(\alpha_{i} x\right) \rightarrow f(\alpha x)$ for all $x \in X$. A functional $f: X \rightarrow R$ is orthogonally additive iff $f(x+y)=f(x)+f(y)$ whenever $x \perp y$. An inner product $\langle\cdot, \cdot\rangle$ on $X$ is compatible when $x \perp y$ iff $\langle x, y\rangle=0$.

THEOREM 1. Let $X$ be an orthogonality vector space. If there exists an $f: X \rightarrow R$ which is even, orthogonally additive, hemicontinuous and not identically 0 then there is a compatible inner product $\langle\cdot, \cdot\rangle$ on $X$. In fact, $\langle x, y\rangle=\frac{1}{4}[f(x+y)-f(x-y)]$ and the corresponding norm satisfies $\|x\|^{2}=$ $f(x)$ or $\|x\|^{2}=-f(x)$ for all $x \in X$.

COROLlary. Let $X$ be an orthogonality vector space and let $f: X \rightarrow R$ be an orthogonally additive, hemicontinuous functional. Then either (1) $f$ is linear or (2) there is a linear functional $f_{2}$ and a compatible inner product with corresponding norm $\|\cdot\|$ such that $f(x)=\|x\|^{2}+f_{2}(x)$ or $f(x)=$ $-\|x\|^{2}+f_{2}(x)$ for all $x \in X$.

If $X$ is a normed space ( $\operatorname{dim} X \geqq 2$ ) in the sequel we shall use James' definition of orthogonality [8]. The following corollary generalizes some of Sundaresan's work [15].

Corollary. Let $X$ be a real normed space, $\operatorname{dim} X \geqq 2$, and let $f: X \rightarrow R$ be orthogonally additive and hemicontinuous. Then either (1) $f$ is linear, or (2) $X$ is an inner product space and there is a linear functional $f_{2}$ and $c \in R$ such that $f(x)=c\|x\|^{2}+f_{2}(x)$ for all $x \in X$.

Since a linear functional is automatically hemicontinuous, hemicontinuity is no restriction on linear functionals. Thus $f$ and $f_{2}$ in the above corollary need not be norm continuous. Our next corollary shows that in an inner product space a bounded orthogonally additive functional is norm continuous and gives a generalization of the Riesz representation theorem.

Corollary. Let $X$ be an inner product space, $\operatorname{dim} X \geqq 2$, and let $f: X \rightarrow R$ be orthogonally additive and satisfy $|f(x)| \leqq M\|x\|$ for all $x \in X$. Then $f$ is a continuous linear functional and hence if $X$ is a Hilbert space there exists $x_{0} \in X$ such that $f(x)=\left\langle x, x_{0}\right\rangle$ for all $x \in X$.

Let $X$ be a real normed space. A functional $f: X \rightarrow R$ is orthogonally increasing iff $x \perp y$ implies $f(x+y) \geqq f(x) ; f$ is radially increasing if $\alpha>1$ implies $f(\alpha x) \geqq f(x)$ for all $x \in X$; and $f$ is spherically increasing if $\|x\|>\|y\|$ implies $f(x) \geqq f(y)$. Decreasing and monotone functionals of these different types are defined in the obvious way. Clearly, spherically increasing implies 
radially increasing and simple examples show that the converse need not hold. In a strictly convex (rotund) normed space, one can show that spherically increasing implies orthogonally increasing but examples can be given which show that this result need not hold in general. In a general normed space, orthogonally increasing need not imply spherically increasing, although as we shall see, this is the case for certain types of normed spaces. An example of an orthogonally increasing functional is the following. Let $g: R^{+} \rightarrow R$, where $R^{+}=$nonnegative reals, be any nondecreasing function and let $f(x)=g(\|x\|)$. In Theorem 2 we characterize orthogonally increasing functionals on a certain type of normed space and show that they are essentially of this form.

Let $X$ be a continuous semi inner product (s.i.p.) space [6]. Giles [6] has shown that a s.i.p. space is continuous iff the norm is Gâteaux differentiable so most of the standard normed spaces are continuous s.i.p. spaces.

Lemma. In a continuous s.i.p. space $X$ with $\operatorname{dim} X \geqq 2$, an orthogonally increasing functional $f$ is radially increasing and also is norm continuous on a dense subset of $X$. Furthermore if $f$ is hemicontinuous at $0 \neq x \in X$ then $f$ is norm continuous at $x$.

We say that a normed space is orthogonally accessible (o.a.) if whenever $\|y\|>\|x\|$ there exists $\lambda \geqq 1$ and $x_{i}, i=1,2, \cdots, n(x, y)$ such that $\lambda x \perp x_{1}$, $\lambda x+\sum_{i=1}^{j-1} x_{i} \perp x_{j}, j=2,3, \cdots, n$, and $y=\lambda x+\sum_{i=1}^{n} x_{i}$. We can show that a large class of normed spaces are o.a. For example, if the two-dimensional cross-sections of the unit sphere satisfy a Lipschitz condition then the normed space is o.a. In particular an inner product space is o.a.

THEOREM 2. Let $X$ be an o.a. continuous s.i.p. space with $\operatorname{dim} X \geqq 2$ and let $f: X \rightarrow R$ be orthogonally increasing. Then $f$ is spherically increasing and there exist a countable number of spheres $s_{1}, s_{2}, \cdots$ such that $f$ is norm continuous at $w$ iff $w \notin \bigcup s_{i}$. Furthermore, there is a nondecreasing function $g: R^{+} \rightarrow R$ such that $f(w)=g(\|w\|)$ for all $w \notin \bigcup s_{i}$.

Corollary. Let $X$ be an o.a. continuous s.i.p. space with $\operatorname{dim} X \geqq 2$ and let $f: X \rightarrow R^{+}$be orthogonally additive. (a) If $X$ is not an inner product space then $f \equiv 0$. (b) If $X$ is an inner product space then there is a $c \in R^{+}$ such that $f(x)=c\|x\|^{2}$ for all $x \in X$.

COROLlaRY. Let $\langle\cdot, \cdot\rangle$ and $\langle\cdot, \cdot\rangle_{1}$ be inner products on a real vector space $X$. If $x \perp y$ implies $x \perp_{1} y$ then there is a $c>0$ such that $\langle u, v\rangle_{1}=$ $c\langle u, v\rangle$ for all $u, v \in X$.

Let $X$ be a real inner product space with $\operatorname{dim} X \geqq 2$. We have already seen that the only solutions to the functional equation $f(x)+f(y)=f(x+y)$ 
whenever $\langle x, y\rangle=0, f(x) \geqq 0$ for all $x \in X$, are functions of the form $f(x)=c\|x\|^{2}, c \geqq 0$. Theorem 2 can be used to solve other functional equations. For example, let $f_{1}: X \rightarrow R$ be the functional $f_{1}(x)=\left(a+\|x\|^{2}\right)^{-1}$ where $a$ is a fixed positive number. If we define

$$
w(a, x, y)=\left(2 a+\|x\|^{2}+\|y\|^{2}\right)^{-1}\left[\left(a+\|y\|^{2}\right) x+\left(a+\|x\|^{2}\right) y\right]
$$

then it is straightforward to verify that $f_{1}$ satisfies the conditions: $f_{1}(x) \geqq 0$ for all $x \in X, f_{1}(x)+f_{1}(y)=f_{1}[w(a, x, y)]$ whenever $\langle x, y\rangle=-a$. Our next theorem shows that these conditions characterize $f_{1}$ up to a constant.

THEOREM 3. A functional $f: X \rightarrow R^{+}$satisfies $f(x)+f(y)=f[w(a, x, y)]$ whenever $\langle x, y\rangle=-a$ iff $f(x)=a f(0)\left(a+\|x\|^{2}\right)^{-1}$.

As a corollary to this theorem we can obtain an important special case of a result due to Gleason [7]. Let $H$ be a Hilbert space and let $P(H)$ denote the set of orthogonal projections on $H$. A state is a map $m: P(H) \rightarrow[0,1]$ satisfying $m(I)=1, m\left(\sum_{i=1}^{\infty} P_{i}\right)=\sum_{i=1}^{\infty} m\left(P_{i}\right)$ if the $P_{i}$ 's are mutually orthogonal and the first sum is in the strong operator topology. We denote the projection onto the one-dimensional subspace generated by a vector $\phi \neq 0$ by $P_{\phi}$.

COROLlaRY. Let $H$ be a real separable Hilbert space of dimension $\geqq 3$ and let $m$ be a state satisfying $m\left(P_{\phi_{0}}\right)=1$ where $\left\|\phi_{0}\right\|=1$. Then $m(P)=$ $\left\langle P_{\phi_{0}}, \phi_{0}\right\rangle$ for all $P \in P(H)$.

\section{REFERENCES}

1. R. V. Chacon and N. Friedman, Additive functionals, Arch. Rational Mech. Anal. 18 (1965), 230-240. MR 30 \#2329.

2. L. Drewnowski and W. Orlicz, On orthogonally additive functionals, Bull. Acad. Polon. Sci. Sér. Sci. Math. Astronom. Phys. 16 (1968), 883-888. MR 39 \#6069.

3. N. Friedman and M. Katz, Additive functionals in $L_{p}$ spaces, Canad. J. Math. 18 (1966), 1264-1271. MR 34 \#6510.

4. - A representation theorem for additive functionals, Arch. Rational Mech. Anal. 21 (1966), 49-57. MR 33 \#245.

5. I. M. Gel'fand and N. Vilenkin, Generalized functions. Vol. 4: Some applications of harmonic analysis, Fizmatgiz, Moscow, 1961; English transl., Academic Press, New York, 1964. MR 26 \#4173; 30 \#4152.

6. J. R. Giles, Classes of semi-inner-product spaces, Trans. Amer. Math. Soc. 129 (1967), 436-446. MR 36 \#663.

7. A. Gleason, Measures on the closed subspaces of a Hilbert space, J. Math. Mech. 6 (1957), 885-893. MR 20 \#2609.

8. R. James, Orthogonality and linear functionals in normed linear spaces, Trans. Amer. Math. Soc. 61 (1947), 265-292. MR 9, 42.

9. H. Leaderman, F. McCrackin and O. Nakada, Large longitudinal retarded elastic deformation of rubberlike network polymers. II. Applications of a general formulation of nonlinear responses, Trans. Soc. Rheology 7 (1963), 111-123. 
10. A. Martin and V. Mizel, A representation theorem for non-linear functionals, Arch. Rational Mech. Anal. 15 (1964), 353-367.

11. V. Mizel, Representation of nonlinear transformations on $L^{p}$ spaces, Bull. Amer. Math. Soc. 75 (1969), 164-168. MR 38 \#4983.

12. V. Mizel and K. Sundaresan, Representation of additive and biadditive functionals, Arch. Rational Mech. Anal. 30 (1968), 102-126. MR 37 \#3341.

13. R. Rivlin, Stress relaxation in elastic materials for varying deformation, Armstrong Cork Co., Technical Report, June 1954.

14. K. Sundaresan, Additive functionals on Orlicz spaces, Studia Math. 32 (1968), 269-276. MR 40 \#1765.

15. - Orthogonality and nonlinear functionals on Banach spaces, Proc. Amer. Math. Soc. 34 (1972), 187-190. MR 45 \#925.

Department of Mathematics, University of Denver, Denver, Colorado 80210 